\title{
Proximicin A, B and C, Novel Aminofuran Antibiotic and Anticancer Compounds Isolated from Marine Strains of the Actinomycete Verrucosispora ${ }^{\dagger}$
}

\author{
Hans-Peter Fiedler, Christina Bruntner, Julia Riedlinger, Alan T. Bull, Gjert Knutsen, \\ Michael Goodfellow, Amanda Jones, Luis Maldonado, Wasu Pathom-aree, \\ Winfried Beil, Kathrin Schneider, Simone Keller, Roderich D. Sussmuth
}

Received: December 21, 2007/Accepted: March 10, 2008

(C) Japan Antibiotics Research Association

\begin{abstract}
A family of three novel aminofuran antibiotics named as proximicins was isolated from the marine Verrucosispora strain MG-37. Proximicin A was detected in parallel in the marine abyssomicin producer "Verrucosispora maris" AB-18-032. The characteristic structural element of proximicins is 4-amino-furan-2carboxylic acid, a hitherto unknown $\gamma$-amino acid. Proximicins show a weak antibacterial activity but a strong cytostatic effect to various human tumor cell lines.
\end{abstract}

Keywords aminofuran antibiotics, antitumor activity, marine actinomycetes, Verrucosispora, physico-chemical properties, proximicin $\mathrm{A}$, proximicin $\mathrm{B}$, proximicin $\mathrm{C}$

\section{Introduction}

The marine environment is proving to be a major source of new natural products including first-in-a-class drug candidates [2] and is the focus of much of our search and discovery programmes. The discovery of novel natural products in marine microorganisms has increased linearly over the past two decades while those found in terrestrial microorganisms have remained almost unchanged over the same period [3]. Recently, we reported on the fermentation, isolation and structure elucidation of abyssomicins $B \sim D$, novel polycyclic polyketide antibiotics from the marine actinomycete "Verrucosispora maris" AB-18-032 which was isolated from sediment collected from the Sea of Japan $[1,4,5]$. The careful evaluation of HPLC chromatograms from extracts of this strain revealed significant amounts of another compound (1) not assignable to any other known compound in our HPLC-UV-Vis database [6]. Remarkably, the extract from another marine member of the rare genus Verrucosispora, strain MG-37, which was isolated from sediment collected in the Raune Fjord (Norway), showed an HPLC-DAD signal identical with compound $\mathbf{1}$ and two further peaks $\mathbf{2}$ and $\mathbf{3}$ with similar UV spectra as $\mathbf{1}$ (Fig. 1), indicating the presence of a family of structurally related peptide metabolites which were named proximicin A (1), B
H.-P. Fiedler (Corresponding author), C. Bruntner, J. Riedlinger: Mikrobiologisches Institut, Universität Tübingen, Auf der Morgenstelle 28, 72076 Tübingen, Germany,

E-mail: hans-peter.fiedler@uni-tuebingen.de

A. T. Bull: Department of Biosciences, University of Kent, Canterbury CT2 7NJ, UK

G. Knutsen: Department of Biology, University of Bergen, Jahnebakken 5, 5020 Bergen, Norway

M. Goodfellow, A. L. Jones, L. A. Maldonado, W. Pathomaree: School of Biology, University of Newcastle, Newcastle upon Tyne NE1 7RU, UK
W. Beil: Institut für Pharmakologie, Medizinische Hochschule Hannover, Carl-Neuberg-Str. 1, 30625 Hannover, Germany

R. D. Süssmuth (Corresponding author), K. Schneider, S. Keller: Institut für Chemie, FG Organische Chemie, Technische Universität Berlin, Straße des 17. Juni 124, 10623 Berlin, Germany, E-mail: suessmuth@chem.tu-berlin.de

${ }^{\dagger}$ Art. No. 42 in 'Biosynthetic Capacities of Actinomycetes'. Art. No. 41: see ref. 1 


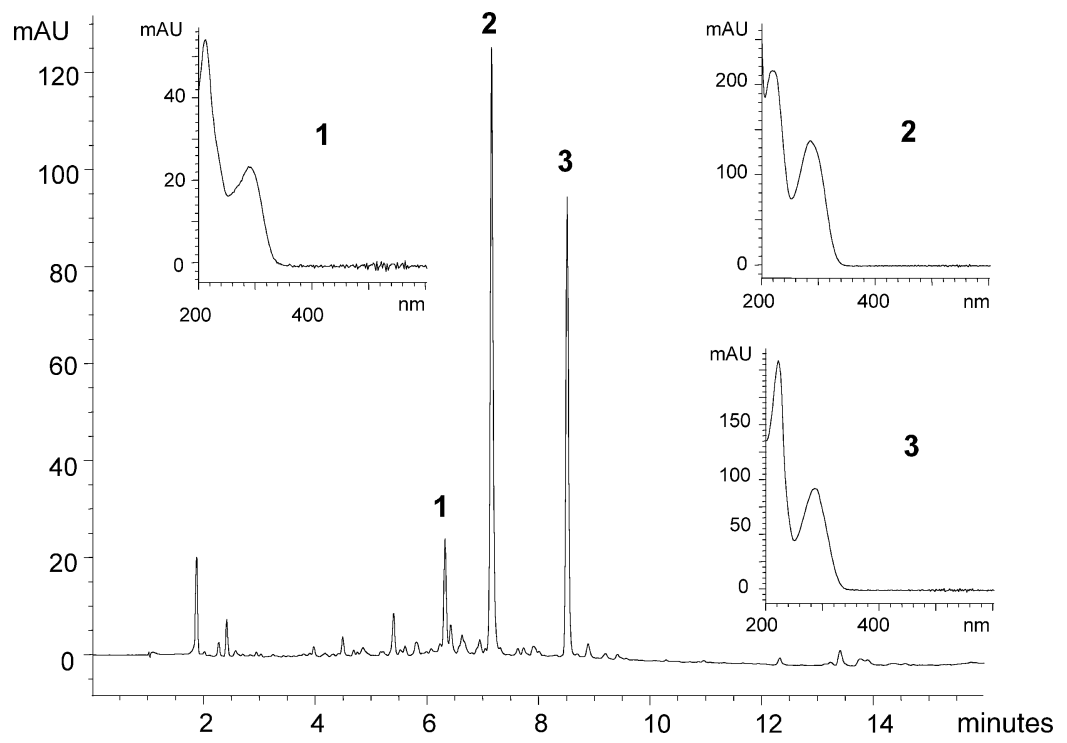

Fig. $1 \mathrm{HPLC}$ analysis of a culture filtrate extract of Verrucosispora strain MG-37 at a fementation time of 96 hours, monitored at $230 \mathrm{~nm}$, and UV-visible spectra of proximicin A (1), B (2) and C (3).

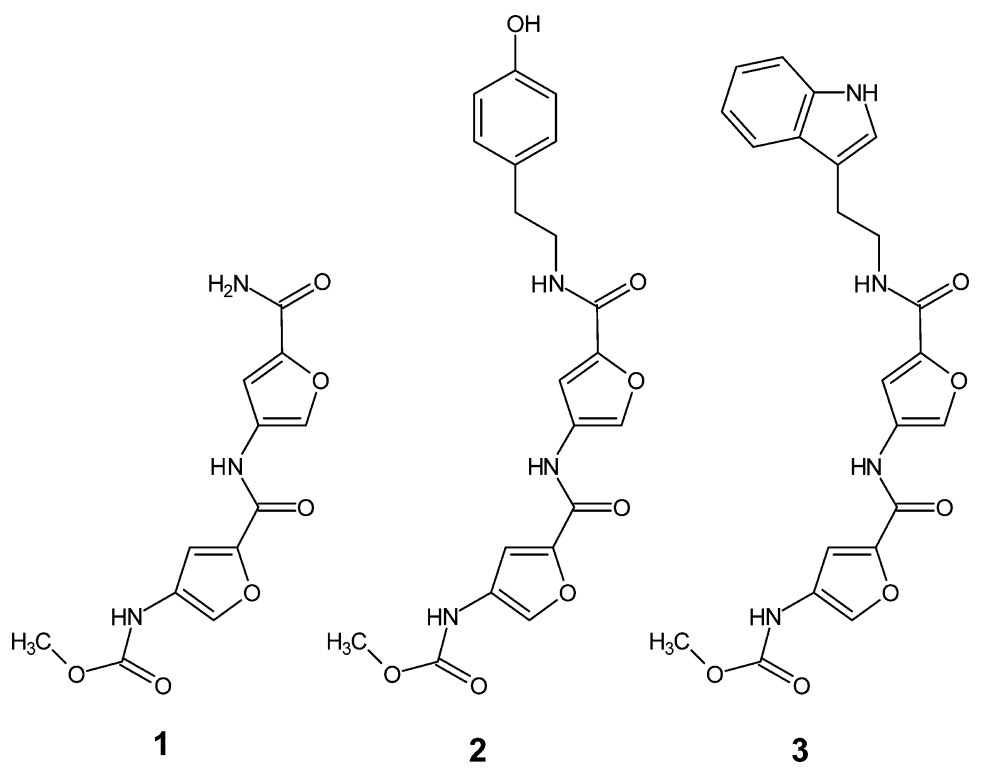

Fig. 2 Structures of proximicin A (1), B (2) and C (3).

(2) and C (3). Herein we report on the taxonomy of the producing strain, the fermentation, isolation, physicochemical properties and biological activities of proximicin $\mathrm{A} \sim \mathrm{C}(\mathbf{1} \sim \mathbf{3})$, bearing 4-amino-furan-2-carboxylic acid as a characteristic structural element, a hitherto unknown $\gamma$ amino acid (Fig. 2).

\section{Materials and Methods}

Producing Organisms and Taxonomy

Strain MG-37 was isolated from sediment collected by us (ATB, GK) in the Raune Fjord, Norway (N 60 15.898 , E $\left.5^{\circ} 08.237\right)$ at a depth of 250 meters. Sediments were collected by box grab from which samples were taken with a sterilized scoop into sterile plastic containers; samples were stored at $4^{\circ} \mathrm{C}$. Strain $\mathrm{AB}-18-032$ was isolated from 
sediment collected from the Sea of Japan at a depth of 289 meters as described previously [4]. The strains were examined for genotypic and phenotypic properties known to be of value in actinobacterial systematics [7].

\section{HPLC-DAD Screening}

The chromatographic system consisted of a HP 1090M liquid chromatograph equipped with a diode-array detector and a HP Kayak XM 600 ChemStation (Agilent Technologies). Multiple wavelength monitoring was performed at 210, 230, 260, 280, 310,360, 435 and $500 \mathrm{~nm}$ and UV-visible spectra measured from 200 to $600 \mathrm{~nm}$. A $10-\mathrm{ml}$ aliquot of the fermentation broth was centrifuged, and the supernatant adjusted to $\mathrm{pH} 4.0$ and extracted with the same volume of EtOAc. After centrifugation, the organic layer was concentrated to dryness in vacuo and resuspended in $1.0 \mathrm{ml} \mathrm{MeOH}$. The mycelial pellet was extracted with $10 \mathrm{ml} \mathrm{MeOH}-\mathrm{Me}_{2} \mathrm{CO}(1: 1)$, the organic extract was filtered, concentrated to dryness in vacuo, and resuspended in $1.0 \mathrm{ml} \mathrm{MeOH} .10-\mu 1$ aliquots of the extracts were injected onto an HPLC column $(125 \times 4.6 \mathrm{~mm})$ fitted with a guard-column $(20 \times 4.6 \mathrm{~mm})$ filled with $5-\mu \mathrm{m}$ Nucleosil-100 C-18 (Maisch, Ammerbuch, Germany). The samples were analysed by linear gradient elution using $0.1 \%$ aq ortho-phosphoric acid as solvent $\mathrm{A}$ and $\mathrm{MeCN}$ as solvent $\mathrm{B}$ at a flow rate of $2.0 \mathrm{ml} /$ minute. The gradient was from 0 to $100 \%$ for solvent $\mathrm{B}$ in 15 minutes with a 2 minute hold at $100 \%$ for solvent B. The UV-visible spectra of the chromatographic peaks were compared with those of 867 reference compounds, mostly antibiotics, stored in our HPLC-UV-Vis database [6].

\section{Fermentation and Isolation}

Batch fermentations of Verrucosispora strain AB-18-032 were performed as described by Riedlinger et al. [4]. Strain MG-37 was cultivated in a 10-liter stirred tank fermentor (Biostat S, B. Braun Melsungen, Germany) in a complex medium that consisted of soluble starch $10 \mathrm{~g}$, glucose $10 \mathrm{~g}$, glycerol $10 \mathrm{~g}$, cornsteep powder (Marcor) $2.5 \mathrm{~g}$, Bacto peptone $5.0 \mathrm{~g}$, yeast extract (Ohly Kat) $2.0 \mathrm{~g}, \mathrm{NaCl} 1.0 \mathrm{~g}$ and $\mathrm{CaCO}_{3} 3.0 \mathrm{~g}$ in 1.0 liter tap water, adjusted to $\mathrm{pH} 7.3$ prior to sterilization. The fermentor was inoculated with 5 vol-\% of shake cultures, grown in 500-ml Erlenmeyer flasks with one baffle for 48 hours on a rotary shaker at $120 \mathrm{rpm}$ and $27^{\circ} \mathrm{C}$ using the same medium. The fermentation was carried out for 96 hours at $27^{\circ} \mathrm{C}$ with an aeration rate of 0.5 liter/liter culture/minute and an agitation of $250 \mathrm{rpm}$.

Hyphlo Super-Cel (2.0\%) was added to the fermentation broth which was separated by multiple sheet filtration into culture filtrate and mycelium. Proximicins were isolated from the culture filtrate (6.3 liters) which was applied to an Amberlite XAD- 16 column $(60 \times 4 \mathrm{~cm})$. Impurities were washed out with water. Proximicin $\mathrm{A}, \mathrm{B}$ and $\mathrm{C}$ were desorbed from the resin by 60,80 and $100 \% \mathrm{MeOH}$, respectively. After concentration to an aq residue, the proximicins were extracted three times with EtOAc. The organic extracts were combined and concentrated in vacuo to dryness. The crude product was dissolved in $\mathrm{CH}_{2} \mathrm{Cl}_{2}$ and subjected to a diol-modified silica gel column $(40 \times 2.6 \mathrm{~cm}$, LiChroprep DIOL, Merck). The separation was accomplished by a linear gradient using $\mathrm{CH}_{2} \mathrm{Cl}_{2}-\mathrm{MeOH}$, starting with $\mathrm{CH}_{2} \mathrm{Cl}_{2}$ and transitioning to $10 \% \mathrm{MeOH}$ within 3 hours at a flow rate of $5.6 \mathrm{ml} /$ minute. Pure proximicins were obtained by Sephadex LH-20 chromatography $(90 \times 2.5 \mathrm{~cm})$ using $\mathrm{MeOH}-\mathrm{CH}_{2} \mathrm{Cl}_{2}(2: 1)$ as the eluent. The proximicins were dissolved in small volumes of tert-BuOH and obtained as white powders after lyophilisation.

\section{Biological Assays}

An agar-plate diffusion assay was used to determine the antibacterial and antifungal spectrum of $\mathbf{1} \sim \mathbf{3}$. Ten $\mu$ of the samples were applied to filter disks ( $6 \mathrm{~mm}$ diameter). The test plates were incubated for 24 hours at a temperature that permitted optimal growth of the test organisms.

The inhibitory activity of $\mathbf{1} \sim \mathbf{3}$ on the growth of tumor cells was tested according to NCI guidelines [8] with human tumor cell lines from gastric adenocarcinoma (AGS), breast carcinoma (MCF 7) and hepatocellular carcinoma (HepG2). Cells were grown in 96-well microtiter plates in 10\% RPMI 1640 medium containing $10 \%$ foetal calf serum in a humidified atmosphere of $5.0 \%$ $\mathrm{CO}_{2}$ in air. Proximicins $(0.1 \sim 10 \mu \mathrm{l} / \mathrm{ml})$ were added to the cells after incubation for 24 hours. Stock solutions were prepared in DMSO; the final DMSO concentration of the cultures was $0.1 \%(\mathrm{w} / \mathrm{v})$. The cells were fixed and cell protein assayed with sulforhodamine B after a 48-hours incubation.

\section{Results}

\section{Taxonomy of the Producing Strains}

A nearly complete $16 \mathrm{~S}$ rRNA gene sequence of strain MG37 was compared with corresponding sequences of representatives of the family Micromonosporaceae. The resultant data showed that the isolate was most closely related to the type strain of Verrucosispora gifhornensis [9]; the two strains share a similarity of $99.6 \%$, a value that corresponds to six nucleotide differences at 1453 locations. The assignment of the strain to the genus Verrucosispora was supported by chemotaxonomic and morphological data 
Table 1 Physico-chemical properties of proximicin A (1), B (2) and C (3)

\begin{tabular}{|c|c|c|c|}
\hline & 1 & 2 & 3 \\
\hline Appearance & White solid & White solid & White solid \\
\hline Molecular weight & 293.1 & 413.1 & 436.1 \\
\hline Molecular formula & $\mathrm{C}_{12} \mathrm{H}_{11} \mathrm{~N}_{3} \mathrm{O}_{6}$ & $\mathrm{C}_{20} \mathrm{H}_{19} \mathrm{~N}_{3} \mathrm{O}_{7}$ & $\mathrm{C}_{22} \mathrm{H}_{20} \mathrm{~N}_{4} \mathrm{O}_{6}$ \\
\hline \multicolumn{4}{|l|}{ ESI-FT-ICR MS (m/z) } \\
\hline Found & $294.07220(\mathrm{M}+\mathrm{H})^{+}$ & $436.11159(\mathrm{M}+\mathrm{Na})^{+}$ & $437.14566(\mathrm{M}+\mathrm{H})^{+}$ \\
\hline Calcd & 294.07206 & 436.11152 & 437.14556 \\
\hline$\cup V \lambda_{\max }^{\mathrm{MeOH}}[\mathrm{nm}]\left(\varepsilon\left[\mathrm{cm}^{2} \mu \mathrm{mol}^{-1}\right]\right)$ & 220 (13.07), 284 (7.62) & 223 (9.35), 285 (5.37) & 222 (42.39), 283 (17.18) \\
\hline \multirow[t]{4}{*}{$\mathrm{IR} v_{\max }\left(\mathrm{cm}^{-1}\right)$} & $3463,3420,3313,3202$ & $3308,3141,3019,2950$ & $3301,3141,3082,3059$, \\
\hline & $3144,2958,2924,2852$, & $2928,2852,1721,1706$ & $2953,2926,2853,1715$, \\
\hline & $1734,1693,1666,1582$ & $1652,1591,1553,1518$ & $1652,1557,1365,1250$ \\
\hline & 1566, 1365, 1249, 1199 & $1367,1244,1200$ & 1199 \\
\hline
\end{tabular}

Table 2 Antibacterial spectrum of proximicin B (2) determined by the agar plate diffusion assay at various concentrations

\begin{tabular}{lccc}
\hline & \multicolumn{3}{c}{ Inhibition zone $(\mathrm{mm})$} \\
\cline { 2 - 4 } Test organism & $1.0 \mathrm{mg} / \mathrm{ml}$ & $0.3 \mathrm{mg} / \mathrm{ml}$ & $0.1 \mathrm{mg} / \mathrm{ml}$ \\
\hline Bacillus subtilis DSM 10 & 12 & - & - \\
Brevibacillus brevis DSM 30 & 22 & 12 & - \\
Staphylococcus aureus DSM 20231 & 12 & - & - \\
\hline
\end{tabular}

as the organism produced whole-organism hydrolysates rich in meso-diaminopimelic acid, contained $\mathrm{N}$-glycolated muramic acid and formed a well developed branched, substrate mycelium which carried single spores with warty ornamentation. In addition, neither aerial hyphae nor sporangia were formed. The two strains shared many phenotypic properties though a range of features can be weighted to distinguish between them. Thus, only strain MG-37 degraded L-tyrosine, grew at $\mathrm{pH} 8.5$ and used erythritol, mannose and sorbose as sole carbon sources for energy and growth.

\section{Fermentation, Isolation and Physico-chemical Properties}

Fermentations of Verrucosispora strain MG-37 reached a maximal biomass of 8 vol- $\%$ after 72 hours of incubation. The production of $\mathbf{1} \sim \mathbf{3}$ started at about 48 hours to reach a maximal amount of $3.0,13$, and $12 \mathrm{mg} /$ liter of $\mathbf{1 , 2}$ and $\mathbf{3}$, respectively after 96 hours of cultivation.

Proximicins were isolated from the culture filtrate by Amberlite XAD-16 chromatography. 1, 2 and 3 were desorbed from the polystyrene resin by 60,80 and $100 \%$ $\mathrm{MeOH}$, respectively. After concentration to an aq residue, the proximicin fractions were extracted with EtOAc and purified by subsequent chromatography on diol-modified silica gel and Sephadex LH-20. The proximicins were obtained as white powders after lyophilization.

The structures of $\mathbf{1} \sim \mathbf{3}$ were elucidated as described by Schneider et al. [10]. The physico-chemical properties are summarized in Table 1. The chemical structures of $\mathbf{1}, \mathbf{2}$ and 3 contained a characteristical furan ring system with a 2,4disubstitution pattern. The structural difference of proximicins lies in the $C$-terminal modifications. $\mathbf{1}$ contains a $C$-terminal amide, whereas $\mathbf{2}$ and $\mathbf{3}$ have tyramine and tryptamine modifications, respectively.

\section{Biological Activity}

The antimicrobial spectra of proximicins were determined by agar plate diffusion assays. Only proximicin B (2) exhibited a moderate growth inhibition of Gram-positive bacteria (Table 2) whereas proximicin C (3) showed a slight growth inhibition only against Brevibaccillus brevis DSM 30. Gram-negative bacteria, such as Escherichia coli K12, Pseudomonas fluorescens DSM 50090, Proteus mirabilis ATCC 35501, yeasts, such as Saccharomyces cerevisiae 
Table 3 Cytostatic activities $(\mu \mathrm{g} / \mathrm{ml})$ of proximicin A (1), B (2) and C (3) against selected human tumor cell lines

\begin{tabular}{|c|c|c|c|c|c|c|}
\hline & \multicolumn{3}{|c|}{$\mathrm{GI}_{50}$} & \multicolumn{3}{|c|}{ TGI } \\
\hline & AGS & HepG2 & MCF 7 & AGS & HepG2 & MCF 7 \\
\hline 1 & 0.6 & 0.82 & 7.2 & $>10^{a}$ & $>10^{d}$ & $>10^{g}$ \\
\hline 2 & 1.5 & 9.5 & 5.0 & $>10^{\mathrm{b}}$ & $>10^{e}$ & $>10^{h}$ \\
\hline 3 & 0.25 & 0.78 & 9.0 & $>10^{c}$ & $>10^{f}$ & $>10^{i}$ \\
\hline
\end{tabular}

$\mathrm{GI}_{50}: 50 \%$ growth inhibition; TGI: $100 \%$ growth inhibition

a $89 \%$ inhibition at $10 \mu \mathrm{g} / \mathrm{ml} ;{ }^{b} 68 \%$ inhibition at $10 \mu \mathrm{g} / \mathrm{ml} ;{ }^{c} 79 \%$ inhibiton at $10 \mu \mathrm{g} / \mathrm{ml} ;{ }^{\text {d }} 96 \%$ inhibition at $10 \mu \mathrm{g} / \mathrm{ml} ;{ }^{\text {e }} 51 \%$ inhibition at $10 \mu \mathrm{g} / \mathrm{ml} ;{ }^{f} 83 \%$ inhibition at $10 \mu \mathrm{g} / \mathrm{ml} ;{ }^{9} 66 \%$ inhibition at $10 \mu \mathrm{g} / \mathrm{ml} ;{ }^{\text {h }} 64 \%$ inhibition at $10 \mu \mathrm{g} / \mathrm{ml} ;{ }^{i} 55 \%$ inhibition at $10 \mu \mathrm{g} / \mathrm{ml}$.

ATCC 9080 and Candida albicans Tü 164, and filamentous fungi, such as Botrytis cinerea Tü 157, Aspergillus viridi nutans CBS 12756 and Penicillium notatum Tü 136, were insensitive to all of the proximicins.

The cytostatic effects of $\mathbf{1} \sim \mathbf{3}$ were tested in different tumor cell lines. All compounds showed significant growth inhibitory activities towards gastric adenocarcinoma (AGS) and hepatocellular carcinoma (Hep G2), whereas breast carcinoma cells (MCF 7) were less sensitive (Table 3).

\section{Discussion}

HPLC-diode array monitoring of extracts from freshly isolated microorganisms is, as we have shown before [11], a powerful technique in the identification of novel secondary metabolites and in this study resulted in the detection of proximicins in two Verrucosispora strains isolated from very different marine locations. It is astonishing that strain AB-18-032, which was isolated from sediment collected in the Pacific Ocean, produced in addition to the PKS type 1-derived abyssomicins the same metabolite, proximicin $\mathrm{A}$, as strain $\mathrm{MG}-37$ which was isolated from a sediment from the Atlantic Ocean. Verrucosispora strain MG-37 does not produce any abyssomicin compounds but exclusively the NRPS-derived polypeptide antibiotics proximicin A, B and C. Conversely, the abyssomicin producing "Verrucosispora maris" AB-18032 produced no proximicin other than proximicin A. These finding reinforce the view that the search for 'rare', in the sense of low abundance, actinomycetes is a propitious route for discovering novel natural drugs. Such searching now is increasingly easy as large strain libraries can be screened rapidly for selected organisms by means of taxon-specific PCR [see ref. 12].
Proximicins show a structural similarity with netropsin and distamycin, pyrrolamidone antibiotics produced by Streptomyces netropsis [13] and "S. distallicus" [14], respectively. These $\gamma$-peptide antibiotics have as characteristic structural element an $N$-methyl-pyrrol ring instead of the furan ring as in the case of proximicins. Pyrrolamidone antibiotics exhibit an antitumor activity caused by a selective binding to AT-rich sequences in the minor groove of DNA [15]. In contrast to netropsin and distamycin, proximicins do not bind to DNA and show a different mode of antitumor action as pyrrolamidone antibiotics, which will be reported in a forthcoming publication.

Acknowledgements RDS acknowledges support from the DFG (project SU 239-5/1) and the European Commission (project ACTINOGEN, 6th Framework, LSHM-CT-2004-005224). ATB and MG acknowledge support from the UK Natural Environmental Research Council (grants NER/T/S/2000/00614 and NER/T/S/ 2000/00616). We thank the University of Bergen, Associate Professor Svein Rune Erga and the crew of the F/F Hans Brattstrom for enabling us to collect samples in Norwegian fjords. The authors thank Georg Grewe and Christian Tänzer, Universität Tübingen, for technical assistance, and Agilent Technologies (Waldbronn, Germany) for HPLC-software support.

\section{References}

1. Keller S, Nicholson G, Drahl C, Sorensen E, Fiedler H-P, Sussmuth RD. Abyssomicins $\mathrm{G}$ and $\mathrm{H}$ and atropabyssomicin $\mathrm{C}$ from the marine Verrucosispora strain AB18-032. J Antibiot 60: 391-394 (2007)

2. Bull AT, Stach JEM. Marine actinobacteria: new opportunities for natural product search and discovery. Trends Microbiol 15: 491-499 (2007) 
3. Schmidt EW. From chemical structure to environmental biosynthetic pathways: navigating marine invertebratebacteria associations. Trends Biotechnol 23: 437-440 (2005)

4. Riedlinger J, Reicke A, Zahner H, Krismer B, Bull AT, Maldonado LA, Ward AC, Goodfellow M, Bister B, Bischoff D, Sussmuth RD, Fiedler H-P. Abyssomicins, inhibitors of the para-aminobenzoic acid pathway produced by the marine Verrucosispora strain AB-18-032. J Antibiot 57: 271-279 (2004)

5. Bister B, Bischoff D, Ströbele M, Riedlinger J, Reicke A, Wolter F, Bull AT, Zähner H, Fiedler H-P, Sussmuth RD. Abyssomicin $\mathrm{C}$ - a polycyclic antibiotic from a marine Verrucosispora strain as an inhibitor of the $p$-aminobenzoic acid/tetrahydrofolate biosynthesis pathway. Angew Chem Int Ed 43: 2574-2576 (2004)

6. Fiedler H-P. Biosynthetic capacities of actinomycetes. 1. Screening for novel secondary metabolites by HPLC and UV-visible absorbance libraries. Nat Prod Lett 2: 119-128 (1993)

7. Tan GYA, Robinson S, Lacey E, Brown R, Kim W, Goodfellow M. Amycolatopsis regifaucium sp. nov., a novel actinomycete that produces kigamicins. Int J Syst Evol Microbiol 57: 2562-2567 (2007)

8. Grever MR, Shepartz SA, Chabner BA. The National Cancer Institute: cancer drug discovery and development program. Semin Oncol 19: 622-638 (1992)

9. Rheims H, Schumann P, Rohde M, Stackebrandt E.
Verrucosispora gifhornensis gen. nov., sp. nov., a new member of the family Micromonosporaceae. Int J Syst Evol Microbiol 48: 1119-1127 (1998)

10. Schneider K, Keller S, Wolter FE, Röglin L, Beil W, Seitz O, Nicholson G, Bruntner C, Riedlinger J, Fiedler H-P, Süssmuth RD. Proximicins A, B and C-antitumor furan analogues of netropsin from the marine actinomycete Verrucosispora. Angew Chem Int Ed, accepted for publication

11. Fiedler H-P, Bruntner C, Bull AT, Ward AC, Goodfellow M, Potterat O, Puder C, Mihm G. Marine actinomycetes as a source of novel secondary metabolites. Antonie van Leeuwenhoek 87: 37-42 (2005)

12. Maldonado LA, Stach JEM, Pathom-aree W, Ward AC, Bull AT, Goodfellow M. Diversity of cultivable actinobacteria in geographically widespread marine sediments. Antonie van Leeuwenhoek 87: 11-18 (2005)

13. Finlay AC, Hochstein FA, Sobin BA, Murphy FX: Netropsin, a new antibiotic produced by a Streptomyces. J Am Chem Soc 73: 341-343 (1951)

14. Arcamone F, Penco S, Orezzi P, Nicolella V, Pirelli A. Structure and synthesis of distamycin A. Nature 203: 1064-1065 (1964)

15. Bailly C, Chaires B, Sequence-specific DNA minor groove binders. Design and synthesis of netropsin and distamycin analogues. Biocon Chem 9: 513-538 (1998) 